\title{
The modecleaner system and suspension aspects of GEO 600
}

\author{
S Gossler ${ }^{1}$, M M Casey ${ }^{3}$, A Freise ${ }^{1}$, H Grote ${ }^{1}$, H Lück $^{1,2}$, P McNamara $^{3}$, \\ M V Plissi ${ }^{3}$, D I Robertson ${ }^{3}$, N A Robertson ${ }^{3}$, K Skeldon $^{3}$, K A Strain ${ }^{3}$, \\ C I Torrie ${ }^{3}$, H Ward ${ }^{3}$, B Willke ${ }^{2}$, J Hough ${ }^{3}$ and K Danzmann ${ }^{1,2}$ \\ ${ }^{1}$ Universität Hannover, Institut für Atom-und Molekülphysik, Abteilung Spektroskopie, \\ Callinstrasse 38, D-30169 Hannover, Germany \\ ${ }_{2}$ Max-Planck Institut für Gravitationsphysik (Albert-Einstein-Institut), Teilinstitut Hannover, \\ Callinstrasse 38, D-30169 Hannover, Germany \\ ${ }^{3}$ Department of Physics and Astronomy, Kelvin Building, University of Glasgow, \\ Glasgow G12 8QQ, UK \\ E-mail: sfg@mpq.mpg.de
}

Received 2 October 2001

Published 18 March 2002

Online at stacks.iop.org/CQG/19/1835

\begin{abstract}
GEO 600 uses two $8 \mathrm{~m}$ triangular ring cavities as a modecleaner system for the stabilization of the laser. To isolate the cavities with respect to the seismic noise the optical components are suspended as double pendulums. The resonances of these pendulums are damped by a local-control loop via magnet-coil actuators acting on the intermediate masses. The suspension scheme and the measured key data (i.e. finesse, linewidth, visibility, throughput and in-lock durations of the cavities, as well as the isolation performance and the resulting frequency stability) of the modecleaner system will be given in this paper. Furthermore an overview of the GEO 600 interferometer suspension will be given.
\end{abstract}

PACS numbers: $0480 \mathrm{~N}, 9555 \mathrm{Y}$

(Some figures in this article are in colour only in the electronic version)

\section{Introduction}

The assembly of the British-German interferometric gravitational wave detector GEO 600 in Ruthe close to Hannover, Germany is nearly completed. All the optical components except for the signal-recyling mirror are already installed and the modecleaner system is optimized to a sufficient state [10].

It is necessary to isolate the gravitational wave detector and the injection optics against the seismic noise, which follows a $10^{-7} \mathrm{f}^{2} \mathrm{~m} \mathrm{~Hz}^{-1 / 2}$ slope above $10 \mathrm{~Hz}$ at the site to meet the sensitivity goals of GEO 600. Furthermore, the Michelson interferometer as well as the 


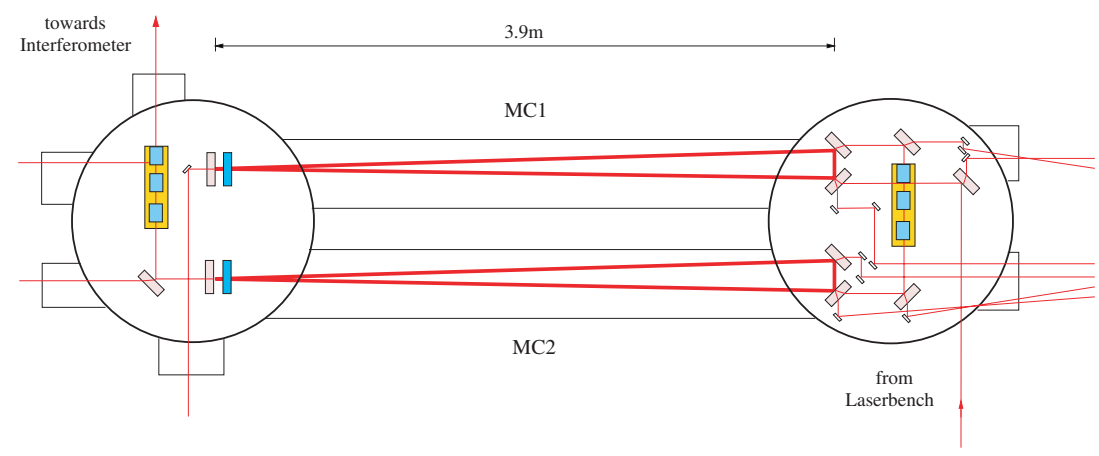

Figure 1. Optical layout of the modecleaner system: 12 similar double pendulums are suspended in two vacuum tanks. Also shown are two double pendulum suspended mounting units, supporting the modulators and Faraday isolators.

modecleaner system needs to be operated in an ultra-high vacuum to avoid contamination of the mirrors and to reduce the effect of fluctuations of the refractive index of the air. Furthermore both acoustic coupling and frictional damping of the suspensions are thereby reduced.

The light source is a $1 \mathrm{~W}$ non-planar ring oscillator (NPRO) from the company Innolight as a master laser and a $14 \mathrm{~W} \mathrm{Nd:YAG} \mathrm{slave} \mathrm{laser} \mathrm{in} \mathrm{bow} \mathrm{tie} \mathrm{configuration} \mathrm{which} \mathrm{is} \mathrm{injection}$ locked to the master $[1,11]$. As a modecleaner system we use two high-finesse cavities in series to obtain the required noise level. The use of a double modecleaner system yields some advantages in spatial (see below) as well as in temporal filtering. At Fourier frequencies corresponding to multiples of the free spectral range of a cavity no temporal filtering is provided. By the use of a system with two cavities of slightly different lengths, these filtering gaps can be avoided.

For seismic isolation we use cascaded pendulums as mechanical low-pass filters, giving a $1 / f^{2}$ isolation performance per pendulum stage above its resonance frequency. All the optical components of the modecleaners are suspended as double pendulums damped at their fundamental resonance frequencies. The main interferometer optics are suspended as triple pendulums plus two vertical stages, as described below.

The suppression of higher order modes of a cavity scales with its finesse $\mathcal{F}$ and depends largely on the geometry of the cavity [7]. As the modecleaner system, GEO 600 uses two high-finesse triangular ring cavities each having two flat mirrors, defining the short side of the triangle, and a curved mirror at the acute angle of the triangle (see figure 1). With that geometry a simplified expression for the suppression factor $S_{m n}$ belonging to the $\mathrm{TEM}_{m n}$ mode, is given by

$$
S_{m n} \simeq \sqrt{1+4 \frac{\mathcal{F}^{2}}{\pi^{2}} \sin ^{2}\left[(n+m) \arccos \sqrt{1-\frac{L}{2 R}}\right]}
$$

where $L$ is the optical pathlength of the cavity. In the case of the modecleaners the optical pathlength $L$ is $8.0 \mathrm{~m}$ for the first cavity and $8.1 \mathrm{~m}$ for the second while the radius of curvature $R$ of the non-planar mirror is $6.72 \mathrm{~m}$. That leads to a ratio of $L / 2 R \simeq 0.6$. With that geometry the suppression factor for the first two higher order modes is approximately $\mathcal{F} / 2$ for one cavity. From former measurements [9] we know that a suppression factor of up to $10^{5}$ may be required. A single cavity would need to have such a high finesse to satisfy this requirement that the throughput would be limited. 
Since the finesses of the two cavities are 2700 and 1900, respectively, the modecleaner system provides a suppression of about six orders of magnitude for the first few higher order modes.

\section{Experimental setup of the modecleaner system}

To suppress the coupling of seismic noise to the optics in the GEO 600 detection window of approximately $50 \mathrm{~Hz}$ to $1 \mathrm{kHz}$, there are different stages of filtering mechanisms. The first stage is formed by passively isolating stacks containing three layers of steel plates separated by two layers of soft rubber cylinders giving a vertical resonance frequency of about $16 \mathrm{~Hz}$ when loaded. The stacks are encapsulated with internally damped convoluted stainless steel bellows to provide the ultra-high vacuum compatibility [5]. On top of the stacks are flex pivots which are very soft in rotation and on top of the flex pivots lies the top plate. The top plate is a hollow stainless steel structure damped from inside with graphite-loaded silicone rubber. All the pendulums in one tank are attached to a common top plate via revolvable tables allowing rotational prealignment of the optical components. The final alignment is done by applying offset currents through the local control coils described below. Both cavities are fully autoaligned with the incoming laser beam with a bandwidth of $0.2 \mathrm{~Hz}$, using the technique of differential wavefront sensing [4].

\subsection{The double pendulums}

For further isolation against seismic noise all the optical components of the two modecleaners are suspended as double pendulums. In addition to the six cavity mirrors four beam steering mirrors are suspended as well as two phase modulators and optical isolators which are mounted on suspended platforms (mounting units).

The intermediate mass forming the first pendulum stage is made of aluminium, has a weight of $0.860 \mathrm{~kg}$ and is suspended from the top plate via two $29.0 \mathrm{~cm}$ long steel wires of $130 \mu \mathrm{m}$ diameter. Onto the intermediate mass two loops of steel wires with a diameter of $102 \mu \mathrm{m}$ are clamped, forming the second $46.0 \mathrm{~cm}$ long stage containing the mirror. The cylindrical mirrors weigh $0.864 \mathrm{~kg}$, are $100 \mathrm{~mm}$ in diameter and $50 \mathrm{~mm}$ thick. Therfore the first resonance frequencies are calculated to be $0.6,1.3,1.6,2.3 \mathrm{~Hz}$ for longitudinal motion and tilt, $0.6,1.5,15,34.5 \mathrm{~Hz}$ for the sideways and roll mode, $0.7,2 \mathrm{~Hz}$ for rotation and 11.8 , $30.1 \mathrm{~Hz}$ for the vertical (or 'bounce') modes (see figure 5). The resonance frequencies of the violin modes of the lower wires are predicted by our MATLAB model to be $195 \mathrm{~Hz}$ and are measured to be in between $190.6 \mathrm{~Hz}$ and $197.1 \mathrm{~Hz}$. From the data shown in figure 2 one can calculate the mechanical quality factor $Q$ of the violin modes to be $5 \times 10^{5}-1 \times 10^{6}$ which is very close to the maximum value that can be obtained with steel wires.

\subsection{The local control}

To suppress the resonant enhancement of the motion of the pendulum we use local control damping with magnet-coil actuators at the intermediate masses. The feedback is applied at the uppermost pendulum stage to benefit from the filtering of the introduced noise by the following pendulum stages. The read-out of the position and movement of the pendulum is done via shadow sensors co-located with the actuator coils in a glass encapsulation. A rigid structure extending from the top plate supports the coils. The motion of the pendulum is monitored and controlled in four degrees of freedom; there is no vertical control. Onto the intermediate mass we bonded four magnets, supporting four 'flags'. While the magnets match 


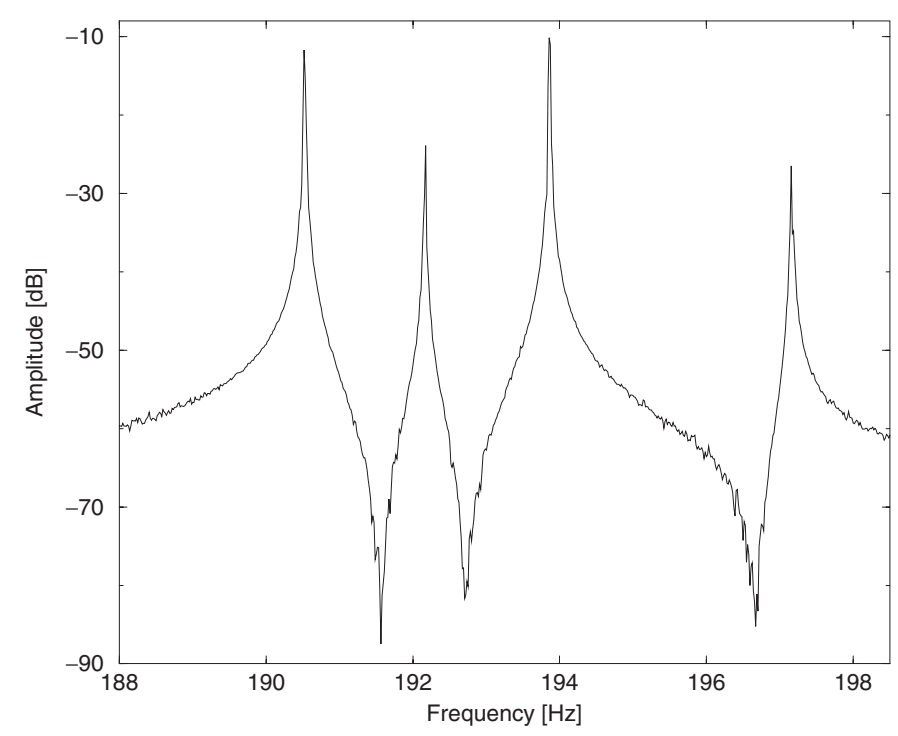

Figure 2. The violin modes of the four steel wires of the lower pendulum stage of the curved mirror of the first modecleaner.

the coils, the flags partly interrupt a beam from an LED detected by a Si photodiode. These simple optical shadow sensors provide a current proportional to the degree the flag penetrates the beam. The range of the coil-magnet actuators is more than $1 \mathrm{~mm}$.

The local control circuit has unity gain frequencies between $0.3 \mathrm{~Hz}$ and $3 \mathrm{~Hz}$. The fact that there is no gain at very low frequencies allows us to apply offset currents to the coils for alignment purposes and to compensate for long-term drifts. The analogue local control servo is digitally supervised and can be controlled/adjusted from a PC using LabView.

\subsection{The length control}

The control scheme of the modecleaner system is given in detail in [3], hence only a short introduction is given here.

To stabilize the laser frequency to the resonance frequency of the first cavity we use the well-known Pound-Drever-Hall sideband technique. Therefore the laser light is phase modulated at 25.2 MHz. The laser frequency is kept on resonance within the first modecleaner with a bandwidth of about $100 \mathrm{kHz}$. This is achieved by using a thermal and a piezo drive acting on the master laser crystal and an EOM as a fast phase shifter. After the first modecleaner the light is phase modulated at $13.0 \mathrm{MHz}$ to stabilize the length of the first modecleaner to the length of the second modecleaner. By controlling the length of the first modecleaner and the laser frequency, a bandwidth of about $25 \mathrm{MHz}$ is realized for this stabilization. Finally, the length of the second modecleaner is stabilized to the length of the power-recycling cavity (2400 $\mathrm{m}$ round trip length).

To control the length of the modecleaners actively it is neccessary to apply feedback at one mirror of each cavity. For that reason we suspended a reaction pendulum separated by a $3 \mathrm{~mm}$ gap from the mirror pendulum to avoid the incoupling of seismic noise through the length control actuators. This reaction pendulum supports three coils matching magnets bonded onto the mirror surface. 


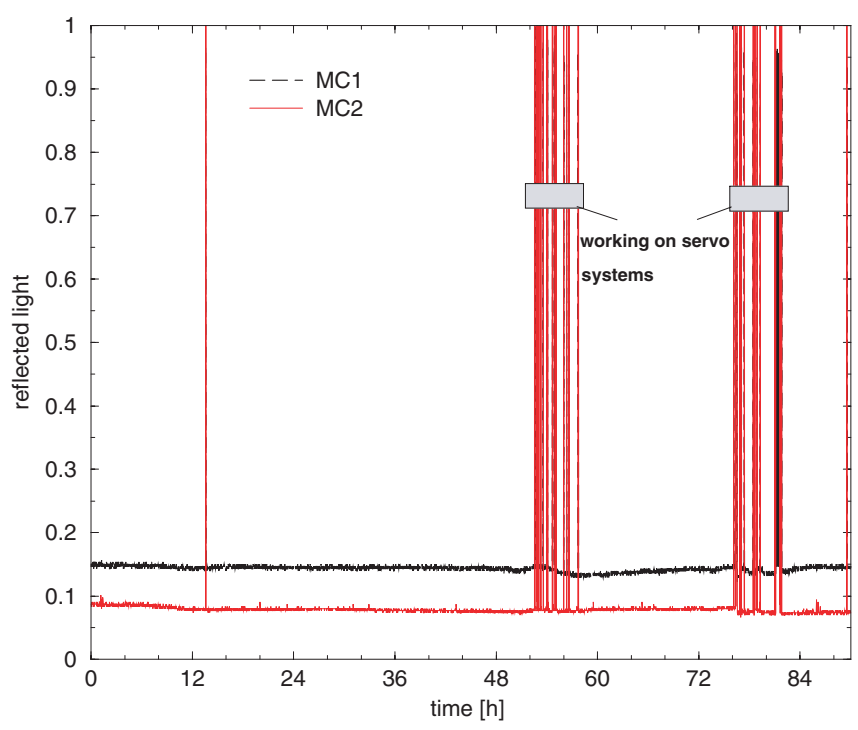

Figure 3. Long-term measurement of the modecleaner's visibilities.

A long-term measurement of the in-lock durations was taken in early 2001. Figure 3 shows a sketch of $90 \mathrm{~h}$ where the visibility of both modecleaners is monitored. During that period the modecleaners fell out of lock only twice, except when people were working on the servo systems. The relock of the cavities is fully automated and can be controlled/adjusted from a LabView PC. The average relock time for the whole system including the slave laser to the master, the master to the first modecleaner and the first modecleaner to the second one is less than $40 \mathrm{~s}$. In order to avoid the excitement of the internal modes of the mirror the modecleaner servos include biquadratic (scultety) filters at 25.9, 35.5, 37.5, 50.5, 53.5, 69.4 and $70.9 \mathrm{kHz}$.

\section{The main suspension}

In contrast to the modecleaner suspensions the main optics of the interferometer are suspended as triple pendulums to obtain a remaining displacement noise of the mirrors of $\mathrm{d} x \simeq 2.4 \times 10^{-20} \mathrm{~m} \mathrm{~Hz}^{-1 / 2}$ at $50 \mathrm{~Hz}$. This corresponds to a seismic noise level which is a factor approximately 3 lower than the expected motion due to thermal noise associated with the internal modes [5].

The triple pendulums are damped in all six degrees of freedom with similar magnet-coil actuators and shadow sensors as desribed above. The two additional actuators control the vertical and roll modes of the pendulum. In addition to the three longitudinal pendulum stages there are two cantilever spring stages improving the vertical isolation (see figure 4). The internal resonances of the upper cantilever springs are damped with magnets and copper tubes acting as eddy current dampers.

The main optics are $180 \mathrm{~mm}$ in diameter, $90 \mathrm{~mm}$ thick and weigh $5.6 \mathrm{~kg}(260 \mathrm{~mm}$, $80 \mathrm{~mm}$ and $9.3 \mathrm{~kg}$ for the beamsplitter). The two upper stages are suspended with steel wires, whereas the third stage is only made of steel wires for the test phase. Finally this stage will be replaced by a monolithic one, providing a higher mechanical quality factor $Q$ of about $5 \times 10^{6}$ for the internal modes of the mirror and $10^{7}$ for the pendulum modes. This leads, 


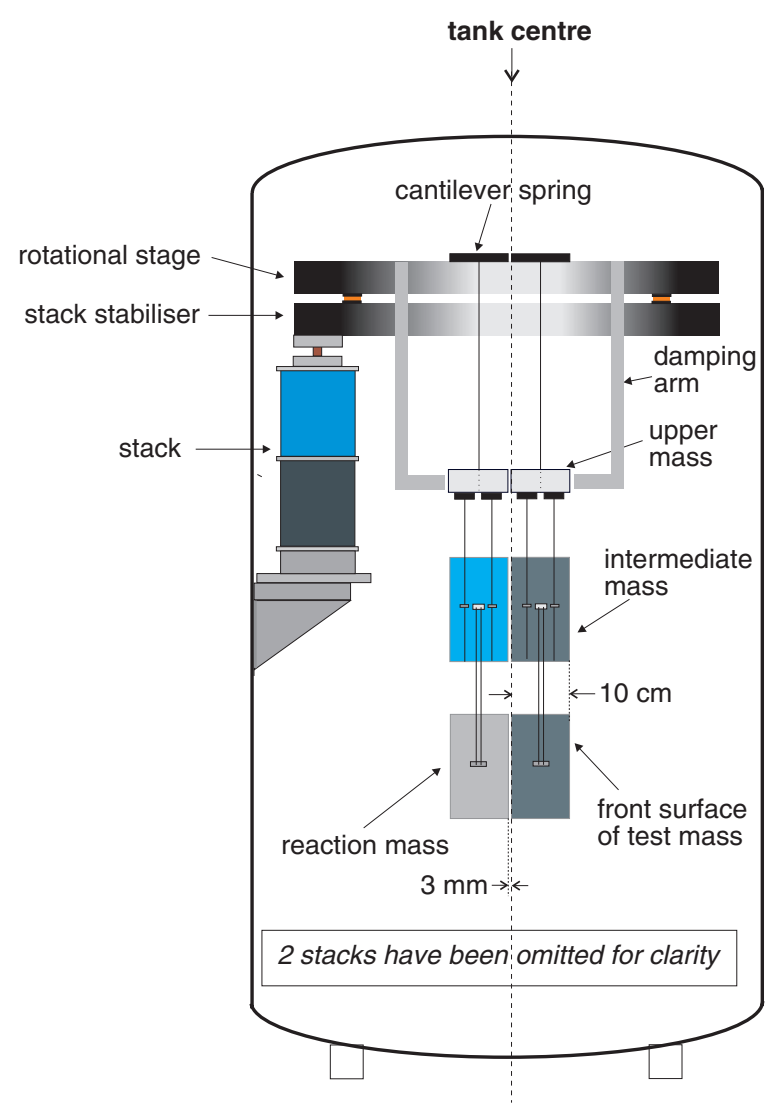

Figure 4. Schematic of a triple pendulum with reaction pendulum.

according to the fluctuation-dissipation theorem, to lower thermally induced fluctuations of the suspension [8]. For internal damping the thermally induced displacement fluctuations are given by

$$
x^{2}(\omega)=\frac{1}{Q} \frac{4 k_{B} T g}{\omega m l\left[\left(\frac{g}{l}-\omega^{2}\right)^{2}+\left(\frac{g}{l}\right)^{2}\left(\frac{1}{Q}\right)^{2}\right]} .
$$

The monolithic suspension consists of the mirror and the intermediate mass, both made of fused silica and four fused silica fibres of diameter $300 \mu \mathrm{m}$ [2]. With the technique of silicate bonding two 'ears' are attached to each mass [6]. Onto these ears the fibres are welded with a hydrogen/oxygen burner such that the two masses and the fibres form a monolithic structure.

It is necessary to damp the $Q$ of the fibres' violin modes to keep the Michelson lock servo stable. For that reason we cover two small regions of the fibres belonging to the first two mechanical eigenmodes with amorphous Teflon dissolved in fluorinert. Two of the five optics are already suspended as monolithic pendulums.

As in the modecleaner case we apply longitudinal feedback via a reaction pendulum suspended $3 \mathrm{~mm}$ behind the mirror pendulum. But at the interferometer suspensions we act on the intermediate pendulum stage with magnet-coil actuators to benefit from the filtering of the introduced noise by the following pendulum stage and to avoid the bonding of magnets onto the mirror which would affect the internal mechanical quality factor. For the higher 


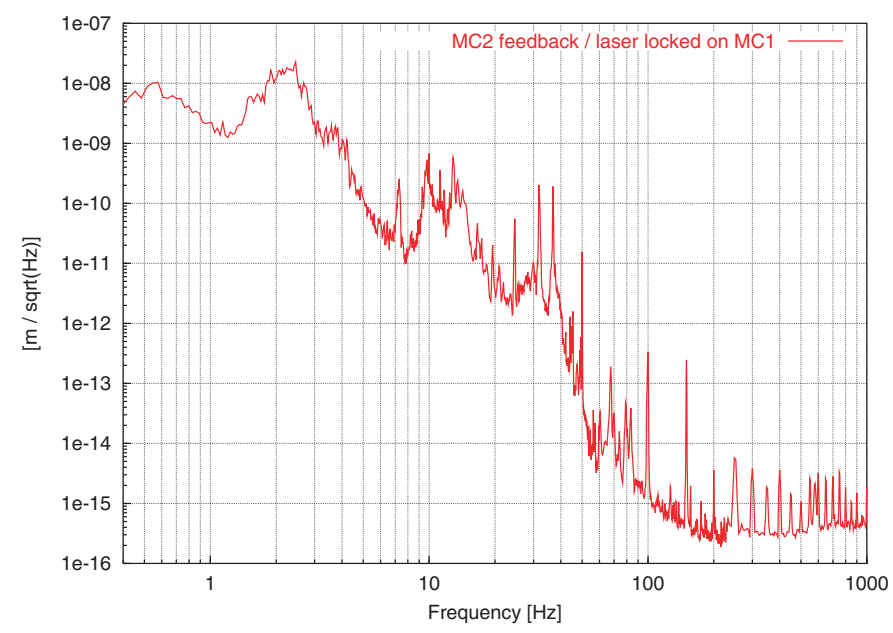

Figure 5. Differential motion of the two modecleaners.

Table 1. Measured modecleaner data.

\begin{tabular}{lll}
\hline & Modecleaner 1 & Modecleaner 2 \\
\hline Finesse & 2700 & 1900 \\
Visibility & $94 \%$ & $92 \%$ \\
Throughput & $80 \%$ & $72 \%$ \\
\hline
\end{tabular}

frequency feedback above about $10 \mathrm{~Hz}$ we use a low noise electrostatic drive at the back of the mirror itself. For that purpose the lowest mass of the reaction pendulum is gold coated with two comb-like interleaved patterns forming a capacitor. The mirror behaves as a dielectric medium in the capacitor field allowing fast feedback by changing the applied voltage.

\section{Results}

The finesse of the modecleaner cavities was measured both with a ringdown and with an amplitude transfer function method to give the values shown in table 1. The measured visibilities and the throughput of each cavity lead to an overall throughput of the whole modecleaner system, including all beam steering mirrors, the phase modulators and optical isolators, of about $50 \%$.

To measure the residual motion of the suspended mirrors we locked both the laser and the second modecleaner to the first modecleaner. From the feedback signal of the second modecleaner one gets the differential motion between the two cavities (see figure 5). In the low-frequency regime up to $1 \mathrm{~Hz}$ the spectrum is dominated by seismically induced motion. The bump at $2.3 \mathrm{~Hz}$ corresponds to the pendulum's longitudinal and tilt modes. The structure at $10-12 \mathrm{~Hz}$ is caused by mechanical resonances of the two tubes which connect the modecleaner vacuum chambers $(11.4$ and $13.2 \mathrm{~Hz}$ ) of the tube that guides the beam from the modecleaners towards the interferometer $(10 \mathrm{~Hz})$ and by a vertical resonance of the double pendulums (common mode at $11.8 \mathrm{~Hz}$ ). The very narrow peak at $23.5 \mathrm{~Hz}$ belongs to a vacuum pump which is now suspended by a coil spring. The two sharp peaks at $31 \mathrm{~Hz}$ and $36 \mathrm{~Hz}$ correspond to the differential vertical mode of the two pendulum stages and to a roll mode. 
The residual differential motion of the two cavities at $100 \mathrm{~Hz}$ is about $10^{-15} \mathrm{~m} \mathrm{~Hz}^{-1 / 2}$. This corresponds to a frequency stability of

$$
\frac{\delta L}{L}=\frac{\delta v}{v} \quad v \simeq 2.82 \times 10^{14} \mathrm{~Hz} \Rightarrow \delta v \simeq 30 \mathrm{mHz} \mathrm{Hz}^{-1 / 2} .
$$

The frequency stability required at the power-recycling mirror of GEO 600 is about $10^{-4} \mathrm{~Hz} \mathrm{~Hz}^{-1 / 2}$ at $100 \mathrm{~Hz}$. This can be achieved by stabilizing the length of the second modecleaner to the length of the power-recycling cavity (2400 $\mathrm{m}$ round-trip length); this is described in detail in [3].

\section{Acknowledgments}

This work was supported by PPARC in the UK, the BMBF in Germany and the state of Lower Saxony, Germany.

\section{References}

[1] Brozek O 1999 Frequenzstabilisierung eines Nd:YAG-Hochleistungs-Laser-Systems für den Gravitationswellendetector GEO 600 PhD Thesis Universität Hannover

[2] Barr B W et al 2002 Proc. of the 4th Edoardo Amaldi Conf. on Gravitational Waves (Perth, Western Australia, 8-13 July 2001) Class. Quantum Grav. 191655

[3] Freise A et al 2002 Proc. of the 4th Edoardo Amaldi Conf. on Gravitational Waves (Perth, Western Australia, 8-13 July 2001) Class. Quantum Grav. 191389

[4] Grote $\mathrm{H}$ et al 2002 Proc. of the 4th Edoardo Amaldi Conf. on Gravitational Waves (Perth, Western Australia, 8-13 July 2001) Class. Quantum Grav. 191849

[5] Plissi M, Torrie C, Husman M, Robertson N, Strain K, Ward H, Lück H and Hough J 2000 Rev. Sci. Instrum. $712539-45$

[6] Rowan S, Twyford S M, Hough J, Gwo D H and Route R 1998 Phys. Rev. Lett. A 246 471-8

[7] Rüdiger A, Schilling R, Schnupp L, Winkler W, Billing H and Maischberger K 1981 A mode selector to suppress fluctuations in laser beam geometry Opt. Acta 28 641-58

[8] Saulson P 1990 Phys. Rev. D 42 2437-45

[9] Skeldon K D and Hough J 1995 Rev. Sci. Instrum. 662760

[10] Willke B et al 2002 Proc. of the 4th Edoardo Amaldi Conf. on Gravitational Waves (Perth, Western Australia, 8-13 July 2001) Class. Quantum Grav. 191377

[11] Zawischa I et al 2002 Proc. of the 4th Edoardo Amaldi Conf. on Gravitational Waves (Perth, Western Australia, 8-13 July 2001) Class. Quantum Grav. 191775 